\title{
ACESSO A MEDICAMENTOS POR VIA JUDICIAL EM UM MUNÍCIPIO DO INTERIOR BAIANO
}

\author{
Gabriella dos Santos Andrade'; Bruno Rodrigues Alencar ${ }^{2}$; \\ 1. Bolsista PIBIC/FAPESB, Graduanda em Farmácia, Universidade Estadual de Feira de Santana, e-mail: \\ gaby_anndrade@hotmail.com \\ 2. Orientador, Departamento de Saúde, Universidade Estadual de Feira de Santana, e-mail: alencarbruno@yahoo.com.br
}

PALAVRAS-CHAVE: Assistência Farmacêutica; Medicamento; Judicialização.

\section{INTRODUÇÃO}

As demandas judiciais por medicamentos para indivíduos com dificuldades no acesso refletem um problema na gestão do SUS, se configurando assim, em um desafio para a gestão da Assistência Farmacêutica (AF) (PEPE; ACÚRCIO, 2014). Trata-se de um fenômeno crescente considerado uma grande ameaça à sanidade financeira e administrativa do SUS, predominando a demanda por medicamentos novos no mercado, de alto custo e alguns ainda patenteados (ASENSI; PINHEIRO, 2015).

Dessa forma, o Poder Judiciário vem interferindo nas políticas públicas de saúde, pois, mesmo que o medicamento não esteja incorporado ao SUS ou que o tratamento já esteja contemplado com outras alternativas terapêuticas, o entendimento é que a instância pública deva fornecer o mesmo (BRITTO, 2016).

Esta interpretação limitada do artigo 196 da Constituição Federal que destaca a saúde como direito de todos e dever do Estado, tornou frequente objeto de interferência do Poder Judiciário, que o traduz como "tudo para todos", gerando litigâncias jurídicas que tem provocado despesas extras na programação financeira do Ministério e Secretarias de Saúde de mais de 500 milhões de reais anuais (BRASIL, 2010).

Neste contexto, esse estudo objetivou analisar como a judicialização interfere na resolubilidade para o acesso a medicamentos do Componente Básico da Assistência Farmacêutica (CBAF) em um município do interior da Bahia.

\section{METODOLOGIA}

1.Tipo de Estudo: trata-se de um estudo de natureza quantitativa.

2. Campo de Estudo: o estudo foi realizado no setor da AF da Secretaria Municipal de Saúde (SMS) de um município do Estado da Bahia.

3. Participantes do Estudo: trata-se de uma pesquisa de análise documental, baseada na coleta de dados secundários contidos nos processos judiciais.

4. Técnicas de Coleta de Dados: a técnica aplicada foi análise documental (MINAYO, 2010), referente a processos judiciais para acesso a medicamentos dos pacientes considerados ativos pelos auxiliares administrativos do setor. Também foram analisados dados referentes a gastos com judicialização nos Relatórios de Gestão Municipal 2013-2016. Os dados coletados foram organizados em um formulário previamente elaborado.

5. Método de Análise de Dados: os dados foram analisados por estatística descritiva, utilizando-se o programa Statistical Package for the Social Sciences (SPSS) versão 20.0.

6. Aspectos éticos: O projeto de pesquisa obedeceu à Resolução no 466/2012 do Conselho Nacional de Saúde (CNS) (BRASIL, 2012), sendo aprovado pelo Comitê de Ética em Pesquisa (CEP) da Universidade Estadual de Feira de Santana (UEFS) sob protocolo ${ }^{\circ}$ $1628201 / 2016$.

\section{RESULTADOS E DISCUSSÃO}

Foram analisadas 181 pastas de pacientes que demandaram judicialmente um total de 393 medicamentos (média de 2,17 por ação). Dentre essas pastas, $85,1 \%(n=154)$ constavam a 
cópia do processo ou da decisão judicial, 32,6\% eram processos dos anos anteriores a 2015, $19,9 \%$ de 2015, 30,9\% de 2016, 5\% até junho de 2017 e, 11,6\% não foi possível identificar o ano.

\section{- Perfil dos requerentes}

Encontrou-se um total de $51,9 \%$ de homens $(n=94)$ e $48,1 \%(n=87)$ mulheres. O requerente de menor idade tinha 2 anos e o mais idoso 82, sendo a média de idade 44 anos. A maioria $(99,4 \%)$ dos requerentes eram residentes do município.

A representação jurídica demonstrou que $80,1 \%(n=145)$ dos usuários tiveram acesso à justiça por advocacia pública via Ministério Público ou Defensoria Pública, $7,7 \% \quad(n=14)$ por advocacia privada e, $12,2 \%(\mathrm{n}=22)$ das pastas não constavam essa informação. Este fato também foi constatado por Aires (2016), no município de Rio Largo - AL nos anos de 2007 a 2014, onde a maior parte das ações $(95,7 \%)$ foi através de representação jurídica pública.

Identificou-se que $41,9 \%$ das prescrições encontradas, eram provenientes do SUS, $27,1 \%$ era de origem privada, $10,5 \%$ de serviços privados conveniados ao SUS. Em 20,4\% das pastas/processos não constavam a prescrição. No estudo de Barreto e outros (2013), no mesmo município, nos anos de 2006 a 2010, 57\% das prescrições foram provenientes de estabelecimentos públicos.

O custo dos medicamentos requeridos variou de $\mathrm{R} \$ 14,02$ a $\mathrm{R} \$ 19.460,00$, sendo em média R \$ 1.413,77 por paciente, considerando para esse cálculo, apenas 70,7\% das pastas $(\mathrm{n}=128)$ que constavam o custo de todos ou de alguns dos medicamentos fornecidos naquele processo.

Dos pacientes considerados ativos, apenas $47 \%(\mathrm{n}=85)$ retiravam regularmente os medicamentos, 40,3\% ( $\mathrm{n}=73)$ foram considerados casos de evasão, ou seja, não retiravam o medicamento há muito tempo, ou não constava nenhuma retirada na pasta; $9,9 \%(\mathrm{n}=18)$ retiravam de forma irregular (com falhas nos períodos); 2,2\% ( $\mathrm{n}=4)$ dos pacientes foram transferidos para o Estado ( $2^{\circ}$ Núcleo Regional de Saúde) ou para a Unidade de Alta Complexidade em Oncologia (UNACON) e, um paciente $(0,6 \%)$ foi a óbito.

\section{- Perfil dos medicamentos e custos das demandas judiciais para o município}

A regularidade sanitária dos medicamentos foi predominante, pois $99,7 \%$ possuíam o registro da Agência Nacional de Vigilância sanitária (ANVISA), com exceção do medicamento tiopronina (Thiola®). Já no estudo de Silva e outros (2017), realizado no Distrito Federal, $17 \%$ dos medicamentos judicializados não possuíam o registro na ANVISA.

Os medicamentos mais solicitados judicialmente, de acordo a classificação do primeiro nível de grupo Anatomical Therapeutic Chemical (ATC) (Tabela 1), pertenciam ao grupo do trato alimentar e metabolismo (26\%), prevalecendo a insulina glargina $(\mathrm{n}=31)$, seguido da insulina glulisina $(n=18)$; sistema nervoso $(18,6 \%)$, com destaque para oxacarbamazepina $(n=11)$ e gabapentina $(n=8)$; e sistema cardiovascular $(16,8 \%)$, destacando o ácido acetilsalicílico $(n=8)$, o succinato de metoprolol $(n=7)$ e a atorvastatina $(n=6)$.

Tabela 1 - Distribuição dos medicamentos demandados judicialmente quanto ao grupo ATC

\begin{tabular}{clcc}
\hline Código & \multicolumn{1}{c}{ Grupo Anatômico } & $\mathbf{n}^{\mathbf{0}}$ & \% \\
\hline A & Trato alimentar e metabolismo & 102 & 26,0 \\
B & Sangue e órgãos formadores de sangue & 34 & 8,7 \\
C & Sistema cardiovascular & 66 & 16,8 \\
$\mathbf{D}$ & Dermatológico & 9 & 2,3 \\
$\mathbf{G}$ & Sistema geniturinário e hormônios sexuais & 19 & 4,8 \\
$\mathbf{H}$ & Hormônios sistêmico, exceto hormônios sexuais e insulinas & 15 & 3,8 \\
$\mathbf{J}$ & Antifecciosos de uso sistêmico & 7 & 1,8 \\
$\mathbf{L}$ & Agentes antineoplásicos e imunomoduladores & 27 & 6,9 \\
\hline
\end{tabular}




\begin{tabular}{clcc}
\hline $\mathbf{M}$ & Sistema músculo esquelético & 21 & 5,3 \\
$\mathbf{N}$ & Sistema nervoso & 73 & 18,6 \\
$\mathbf{P}$ & Produtos antiparásiticos, inseticidas e repelentes & 1 & 0,3 \\
$\mathbf{R}$ & Sistema respiratório & 6 & 1,5 \\
$\mathbf{S}$ & Órgãos sensoriais & 12 & 3,1 \\
$\mathbf{V}$ & Vários (não enquadrados em classe terapêutica específica) & 1 & 0,3 \\
Total & & 393 & 100 \\
\hline
\end{tabular}

Fonte: Autora.

Dos medicamentos solicitados judicialmente, 66,2\%, não pertenciam às listas de medicamentos do SUS. Entre os que faziam parte da Relação Nacional de Medicamentos Essenciais (RENAME) 20,4\% pertenciam ao CBAF (Figura 1).

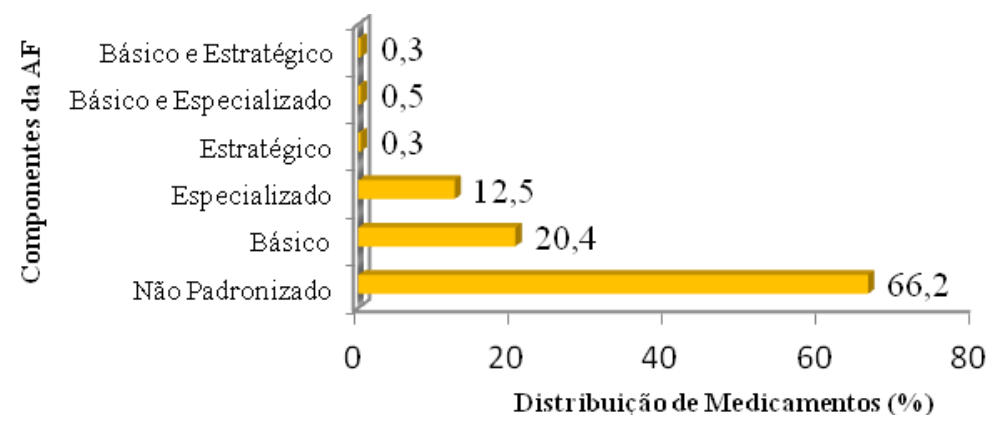

Figura 1- Distribuição de medicamentos padronizados no SUS

No estudo de Barreto e outros (2013) realizado nesse mesmo município, entre 2006 e 2010, a maioria dos medicamentos solicitados judicialmente não pertenciam ao elenco dos programas de AF do SUS, mas dentre os incluídos na lista municipal, prevaleceu do CBAF, com 20,3\% dos medicamentos. Este fato revela que há desabastecimento no município, já que são demandados medicamentos que deveriam estar disponíveis nas unidades de saúde. Borges e outros (2015) apontaram que a cada quinzena, havia em média um desabastecimento de $34,28 \%$ dos itens da lista municipal nas Unidades de Saúde da Família.

Os medicamentos da lista municipal demandados judicialmente foram: ácido acetilsalicílico, lidocaína, diazepam, sinvastatina, insulinas (nph e regular), prednisona, ciprofloxacino, ácido fólico, clonazepam, fluoxetina, anlodipino, omeprazol, hidroclorotiazida, losartana, ibuprofeno, dipirona, amitriptilina, carbamazepina, entre outros.

Os gastos judiciais têm prejudicado o financiamento do SUS, sendo esse problema comprovado em vários estados e cidades do Brasil (FREITAS, 2014). No município estudado não é diferente, conforme apontam os dados dos Relatórios de Gestão Municipal (Quadro 1):

Quadro 1: Gastos com o CBAF e com os medicamentos judiciais do munícipio

\begin{tabular}{|c|c|c|}
\hline Ano & Medicamentos Judiciais (em reais R\$) & CBAF (em reais R\$) \\
\hline 2013 & $531.920,46$ & $4.190 .985,42$ \\
\hline 2014 & $397.552,01$ & $2.873 .560,00$ \\
\hline 2015 & $1.054 .313,80$ & $5.892 .205,88$ \\
\hline 2016 & $969.734,96$ & $7.383 .453,45$ \\
\hline Total & $2.953 .521,23$ & $20.340 .204,75$ \\
\hline
\end{tabular}

Fonte: BAHIA. Prefeitura Municipal. Relatórios de Gestão. Secretaria Municipal de Saúde.

Os custos com a aquisição de medicamentos para as demandas judiciais nos anos de 2013 a 2016 atingiram aproximadamente 3 milhões de reais. Verifica-se assim, que o acesso a medicamentos por essa via é impactante para o sistema de saúde, por esse não dispor de recursos planejados e destinados para esse gasto, comprometendo muitas vezes outros programas e políticas de saúde. 
Além disso, 40,3\% dos pacientes foram considerados casos de evasão e, 9,9\% faziam a retirada de forma irregular, revelando assim, a falta de controle nos processos e de acompanhamento dos usuários, sendo, portanto perceptível o uso irracional de medicamentos e a má utilização dos recursos públicos para fins individuais e não coletivos.

\section{CONSIDERAÇÕES FINAIS}

Os custos das demandas judiciais por medicamentos no período compreendido entre os anos de 2013 a 2016 somaram aproximadamente 3 milhões de reais, trazendo impacto no orçamento municipal. Estes gastos interferem na aquisição de medicamentos do CBAF, uma vez que o município poderia ampliar ainda mais a sua contrapartida.

A maioria $(66,2 \%)$ dos medicamentos demandados judicialmente não são padronizados pelo SUS, entretanto, 20,4\% dos mesmos pertenciam ao CBAF, revelando possíveis falhas na gestão da AF. Além disso, alguns fatores organizacionais podem contribuir para o aumento destes custos, como a falta de informações cadastrais dos pacientes, e ausência de acompanhamento farmacoterapêutico dos pacientes que recebem os medicamentos sem orientação quanto ao uso correto, efeitos farmacológicos previstos, sejam esses favoráveis ou adversos.

A intervenção judicial pode ser indiretamente resolutiva para quem tem acesso à informação e à justiça e utiliza esta via alternativa, de forma individual, para garantir o acesso ao medicamento. Contudo, considerando os princípios do SUS, esta via infringe os princípios da integralidade e da equidade, desorganiza as normas e responsabilidades previstas nas portarias ministeriais que definem os componentes da $\mathrm{AF}$, além de impor aos municípios custos elevados com a aquisição de medicamentos de forma emergencial.

\section{REFERÊNCIAS}

ASENSI, F. D.; PINHEIRO, R. (Coords.). Judicialização da saúde no Brasil: dados e experiência. Brasília: Conselho Nacional de Justiça, 2015.

AIRES. C. C. N. F. Perfil das demandas judiciais para o fornecimento de medicamentos no município de Rio Lago-AL. Dissertação de Mestrado. Universidade Federal de Alagoas. Maceió, 2016.

BARRETO, J. L. et. al. Perfil das demandas judiciais por medicamentos em municípios do Estado da Bahia. Rev. Baiana de Saúde Pública, Salvador, v.37, n.3, p.536-552, 2013.

BORGES, D. S. et al. Disponibilidade de medicamentos essenciais em uma unidade de saúde da família de Feira de Santana - BA. J. Bras. Econ. Saúde, v.7, 2015.

BRASIL. Ministério da Saúde. Da excepcionalidade às linhas de cuidado: o Componente Especializado da Assistência Farmacêutica. Brasília- DF: Ministério da Saúde, 2010. 262p . Conselho Nacional de Saúde. Resolução nº466, de 12 de dezembro de 2012.

Brasília, DF: Conselho Nacional de Saúde, 2012.

BRITTO, A. L. S. A judicialização do acesso a medicamentos no SUS da Bahia.

Dissertação (Mestrado). Faculdade de Direito-Universidade Federal da Bahia, Salvador, 2016.

FREITAS, C. L. T. Judicialização da saúde, solidariedade e ressarcimento: destaques da posição dos estados frente à polarização de entendimentos. In: SANTOS, L.; TERRAZAS, F. (Org.). Judicialização da saúde no Brasil. Campinas - SP: Saberes Editora, 2014. p. 60-93. MINAYO, M. C. S. O desafio do conhecimento: Pesquisa qualitativa em saúde. 12 ed. São Paulo: Hucitec/Abrasco, 2010.

PEPE, V. L. E.; ACÚRCIO, F. A. A.; Assistência Farmacêutica e Demandas Judiciais de Medicamentos no Sistema Único de Saúde. In: CASTRO, C. G. S. O.; et al. Assistência Farmacêutica: gestão e prática para profissionais da saúde. Rio de Janeiro: FIOCRUZ, 2014. SILVA, E. M.; ALMEIDA, K. C.; PESSÔA, G. S. C. Análise do gasto com judicialização de medicamentos no Distrito Federal, Brasil. Cad. Ibero-Amer. Dir. Sanit., Brasília, v. 6, 2017. 